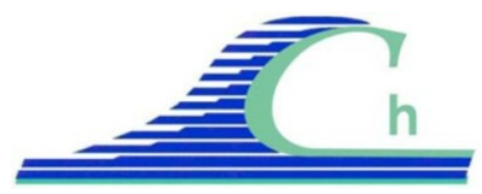

XII ${ }^{\text {èmes }}$ Journées Nationales Génie Côtier - Génie Civil

Cherbourg, 12-14 juin 2012

DOI:10.5150/jngcgc.2012.052-T C Editions Paralia CFL

disponible en ligne - http://www.paralia.fr - available online

\title{
Modélisation de l'effet de la direction des vagues sur la dynamique de barres en croissant
}

\author{
Jérôme THIEBOT ${ }^{1}$, Roland GARNIER ${ }^{2}$, \\ Albert FALQUES ${ }^{3}$, Sylvain GUILLOU ${ }^{1}$
}

1. Laboratoire Universitaire des Sciences Appliquées de Cherbourg (LUSAC), EA4253, Site Universitaire, BP78, 50130 Cherbourg-Octeville, France.

jerome.thiebot@unicaen.fr ; sylvain.guillou@unicaen.fr

2. Instituto de Hidraulica Ambiental IH Cantabria, Universidad de Cantabria, PCTCAN, C/ Isabel Torres 15, 39011 Santander, Espagne.

roland.garnier@unican.es

3. Departament de Fisica Aplicada, Universitat Politècnica de Catalunya (UPC), C/ Jordi Girona 1-3, E-08034-Barcelona, Espagne.

falques@fa.upc.edu

\section{Résumé :}

Les modèles morphodynamiques de plages permettent de simuler l'apparition de figures sédimentaires rythmiques par auto-organisation. Les approches les plus classiques consistent à simuler la formation de barres en croissant par auto-organisation à partir d'une barre rectiligne. En nature, ce type d'évolutions morphodynamiques se produit pendant les séquences down-state selon la classification de WRIGHT \& SHORT (1984). Les mécanismes contrôlant la disparition des figures rythmiques pendant les séquences up-state sont mal compris aujourd'hui et les modèles actuels n'arrivent pas à les reproduire correctement. Dans cet article, nous étudions l'influence du courant longshore sur la disparition des figures rythmiques. Le modèle utilisé est appelé MORFO55. C'est un modèle 2DH non linéaire. Une configuration de plage idéalisée avec une barre parallèle à la côte est considérée. La phase préliminaire des simulations consiste à former des barres en croissant par auto-organisation (i.e. évolution observée pendant les séquences down-state). Une fois que les barres croissant sont formées et qu'un équilibre dynamique est atteint, la direction des vagues au large est modifiée pour engendrer un courant parallèle à la plage. Dans les configurations testées, l'apparition d'un courant longshore est rapidement suivie par la disparition des figures rythmiques. Il semble donc que les courants parallèles à la plage peuvent jouer un rôle significatif sur la disparition des barres en croissants pendant les séquences up-state. La réponse du système est analysée afin de préciser les mécanismes contrôlant ces évolutions.

\section{Mots-clés :}

Barres sableuses - Modélisation - Morphodynamique - Auto-organisation - Sédiments 


\section{Introduction}

Les plages exposées aux vagues sont souvent caractérisées par la présence de barres sableuses parallèles à la côte le long desquelles peuvent apparaître des figures rythmiques telles que les barres en croissant, les barres-baïnes, les barres transverses... D'après le schéma séquentiel de WRIGHT \& SHORT (1984), une configuration de plage à forte pente avec un point de déferlement situé près du bord (sans barre parallèle) est dite réflective. Une plage peu pentue, qui, soumise à la houle, présente une zone de surf étendue vers le large (barre parallèle peu prononcée, nombreux points de déferlement) est dite dissipative. Le passage d'une configuration de plage réflective (dissipative) à une configuration dissipative (réflective) est appelé séquence "up-state" ("down-state"). Cette séquence se traduit par une succession de configurations intermédiaires comportant des structures rythmiques évoquées précédemment. Une séquence down-state est caractérisée par une lente migration vers la côte de la barre parallèle, qui, par ailleurs, évolue d'une structure rectiligne à une structure rythmique. Une séquence up-state est caractérisée par la migration vers le large de la barre, mais aussi par la disparition de ces figures rythmiques. Dans cet article, nous nous intéressons aux mécanismes contrôlant la formation et la disparition des figures rythmiques en relation avec les séquences down-state et up-state, respectivement.

La plupart des modèles morphodynamiques permettent de reproduire l'apparition de figures rythmiques le long des barres par auto-organisation pendant les séquences downstate (FALQUES et al., 2000, CALVETE et al., 2005, GARNIER et al., 2010, CASTELLE \& RUESSINK, 2011). Il s'agit d'un mécanisme d'instabilité : une modification du fond modifie l'hydrodynamique qui modifie le transport sédimentaire qui modifie à son tour le fond et ainsi de suite. En revanche, les mécanismes contrôlant la disparition des figures rythmiques pendant les séquences up-state sont encore mal connus. A notre connaissance, il n'existe aucun modèle numérique capable de reproduire correctement les séquences up-state. Des observations récentes suggèrent que la présence d'un courant longshore induit par des vagues de direction oblique favorise la disparition de figures rythmiques (PRICE \& RUESSINK, 2011). Les courants longshore permettraient donc d'expliquer partiellement la disparition des figures rythmiques pendant les séquences up-state. L'objet de cet article est de tester cette hypothèse avec un modèle d'auto-organisation non linéaire nommé MORFO55 (CABALLERIA et al., 2002, GARNIER et al., 2006).

\section{La Méthode}

\subsection{Le modèle MORFO55}

MORFO55 (CABALLERIA et al., 2002, GARNIER et al., 2006, GARNIER et al., 2008, GARNIER et al., 2010) est un modèle 2DH basé sur une approche à phase moyennée. Le système d'équations utilisées repose sur la conservation de la masse (1), 


\section{XII ${ }^{\text {èmes }}$ Journées Nationales Génie Côtier - Génie Civil \\ Cherbourg, 12-14 juin 2012}

de la quantité de mouvement (2) et du sédiment (3). La propagation des vagues est modélisée avec l'équation de conservation de la densité d'énergie des vagues (4) et la loi de SNELL.

$$
\begin{aligned}
& \frac{\partial D}{\partial t}+\frac{\partial}{\partial x_{i}}\left(D v_{i}\right)=0 \\
& \frac{\partial v_{i}}{\partial t}+v_{j} \frac{\partial v_{i}}{\partial x_{j}}=-g \frac{\partial z_{s}}{\partial x_{i}}-\frac{1}{\rho D} \frac{\partial}{\partial x_{j}}\left(S_{i j}^{\prime}-S_{i j}^{\prime \prime}\right)-\frac{\tau_{b i}}{\rho D} \\
& \frac{\partial z_{b}}{\partial t}+\frac{1}{1-p} \frac{\partial q_{j}}{\partial x_{j}}=0 \\
& \frac{\partial E}{\partial t}+\frac{\partial}{\partial x_{j}}\left(\left(v_{j}+c_{g j}\right) E\right)+S_{i j}^{\prime} \frac{\partial v_{j}}{\partial x_{j}}=-\varepsilon
\end{aligned}
$$

où $z_{s}$ et $z_{b}$ sont les niveaux de la surface libre et du fond, $D$ est la hauteur d'eau moyenne $\left(D=z_{s}-z_{b}\right), t$ est le temps, $v$ est le vecteur vitesse moyenné sur la verticale $\left(v=\left(v_{1}, v_{2}\right)\right)$, $g=9,81 \mathrm{~ms}^{-2}$ est l'accélération de la gravité, $\rho=1024 \mathrm{kgm}^{-3}$ est la masse volumique de l'eau. $S_{i j}^{\prime}$ et $S_{i j}^{\prime \prime}$ sont les tenseurs des contraintes de radiation et de Reynolds, $\vec{\tau}_{b}$ est la contrainte de frottement au fond (MEI, 1989), $p=0,4$ est la porosité du sédiment, et $q_{j}$ sont les deux composantes du vecteur flux horizontal de sédiment $\vec{q}$. $E$ est la densité d'énergie, $\vec{c}_{g}$ est le vecteur vitesse de groupe et $\varepsilon$ est le taux de dissipation induit par le déferlement et le frottement au fond. Le flux de sédiment horizontal (5) est basé sur la formule de SOULSBY \& VAN RIJN (6) :

$$
\begin{aligned}
& \vec{q}=\alpha\left(\vec{v}-\mu_{b} \vec{\nabla} h\right) \\
& \alpha=A\left[\left(|\vec{v}|^{2}+\frac{0.018}{c_{D}} u_{b}^{2}\right)^{1 / 2}-u_{\text {crit }}\right]^{2.4} \operatorname{si}\left(|\vec{v}|^{2}+\frac{0.018}{c_{D}} u_{b}^{2}\right)^{1 / 2}>u_{\text {crit }} \\
& \text { sinon }
\end{aligned}
$$

où $\alpha$ est un coefficient issu de SOULSBY (1997), $h$ est l'amplitude des perturbations du fond (écart par rapport à la bathymétrie initiale), $\gamma$ est un coefficient traduisant l'effet de pente, $u_{b}$ est l'amplitude de la vitesse orbitale $\mathrm{rms}$ au niveau du fond, $A$ est un coefficient dépendant des caractéristiques du sédiment $\left(d_{50}=200 \mu \mathrm{m}\right)$ et de la profondeur $D, c_{D}$ est un coefficient de frottement dépendant de la rugosité du fond $z_{0}$ et de la profondeur $\left(c_{D}=\left(0.4 /\left(\ln \left(D / z_{0}\right)-1\right)\right)^{2}\right)$, et $u_{c r i t}$ est la vitesse critique d'érosion. Le pas de temps hydrodynamique est $0,05 \mathrm{~s}$. Le pas de temps morphodynamique est de $5 \mathrm{~s}$. Les mailles mesurent $5 \mathrm{~m}$ et $10 \mathrm{~m}$ dans les directions perpendiculaire et parallèle à la plage respectivement. Ces paramètres de discrétisation en temps et en espace, issus de GARNIER et al. (2008), permettent à la fois d'assurer la stabilité numérique du modèle et de décrire avec une précision suffisante la morphologie des figures rythmiques. Des 
mailles plus petites conduisent à des résultats comparables (si le critère de stabilité du modèle reste vérifié). Des mailles plus grandes ne permettent pas de décrire correctement la géométrie des barres en croissant.

\subsection{La configuration modélisée}

La plage modélisée comporte une barre parallèle à la plage. Le profil est représentatif de la plage de Duck (Caroline du Nord). La formulation est celle de YU \& SLINN (2003) :

$$
z_{b}(x)=-a_{0}-a_{1}\left(1-\frac{\beta_{2}}{\beta_{1}}\right) \tanh \left(\frac{\beta_{1} x}{a_{1}}\right)-\beta_{2} x+a_{2} \exp \left(-5\left(\frac{x-x_{c}}{x_{c}}\right)^{2}\right)
$$

où $a_{0}=25 \mathrm{~cm}, a_{1}=2,97 \mathrm{~m}, a_{2}=1,5 \mathrm{~m}, \beta_{1}=0,075, \beta_{2}=0,0064$ et $x_{c}=80 \mathrm{~m}$.

Dans la direction parallèle à la plage (perpendiculaire) le domaine de calcul mesure 2 $\mathrm{km}(250 \mathrm{~m})$. La profondeur sur la limite offshore du domaine est de 4,5 m. Des perturbations aléatoires (bruit blanc) d'amplitude $5 \mathrm{~cm}$ sont imposées au début de la simulation pour initier l'auto-organisation du système. Les vagues sur la frontière du large ont une hauteur rms de $1 \mathrm{~m}$ et une période pic de $6 \mathrm{~s}$. Plusieurs incidences ont été imposées sur la frontière du modèle pour pouvoir étudier la réponse du système pour différentes vitesses de courant longshore. Au niveau de la crête de la barre, les angles d'incidence testés sont : $\theta=\mathrm{O}^{\circ}, 3,5^{\circ}, 7^{\circ}$ et $13,5^{\circ}$. Des conditions latérales périodiques sont utilisées.

\subsection{Outils d'analyse}

A partir des équations (1), (3) et (5), FALQUES et al. (2000) ont introduit l'équation d'évolution $\mathrm{du}$ fond appelée Bottom Evolution Equation (BEE). Nous utilisons l'approximation de GARNIER et al. (2008) :

$$
\frac{\partial h}{\partial t} \approx-D \vec{v} \cdot \vec{\nabla} C+\vec{\nabla} \cdot(\Gamma \vec{\nabla} h) \text { avec } \Gamma=\alpha \gamma u_{b} \text { et } C=\alpha / D
$$

L'équation (8) est utile pour comprendre la réponse morphodynamique du système à une configuration hydrodynamique donnée. D'après GARNIER et al. (2010), le premier terme du membre de droite $(-D \vec{v} \cdot \vec{\nabla} C)$ est un terme de production qui est responsable de la croissance ou de l'amortissement des instabilités. Le second terme $(\vec{\nabla} \cdot(\Gamma \vec{\nabla} h))$ est diffusif et traduit les effets de pente. Pour estimer les contributions des différents termes, GARNIER et al. (2006) utilisent l'analyse globale qui consiste à intégrer les termes de l'équation (8) sur l'ensemble du domaine. Ainsi, la production $P$ et la diffusion $\Delta$ sont estimées par les relations (9). Le taux de croissance des instabilités et la norme euclidienne des perturbations sont calculés avec les relations (10).

$$
P=-\frac{1}{L_{x} L_{y}} \int_{0}^{L_{y}} \int_{0}^{L_{x}} h D \vec{v} \cdot \vec{\nabla} C d x d y \quad \Delta=-\frac{1}{L_{x} L_{y}} \int_{0}^{L_{y}} \int_{0}^{L_{x}} h \vec{\nabla} \cdot(\Gamma \vec{\nabla} h) d x d y
$$

où $L_{x}$ et $L_{y}$ sont les dimensions du domaine dans les directions perpendiculaire et parallèle à la plage, respectivement. 
$\sigma=\frac{1}{\|h\|^{2}}(P-\Delta)$

$$
\|h\|=\left(\frac{1}{L_{x} L_{y}} \int_{0}^{L_{y}} \int_{0}^{L_{x}} h^{2} d x d y\right)^{1 / 2}
$$

\section{Les résultats}

\subsection{Formation des barres en croissants}

La phase préliminaire des simulations consiste à former des croissants par autoorganisation à partir d'une barre rectiligne parallèle à la côte. Il s'agit ici de l'évolution observée pendant les séquences down-state. Pour cette phase, nous avons imposé des vagues normales à la côte $\left(\theta=0^{\circ}\right)$. L'évolution temporelle des variables $\|h\|, P, \Delta$ et $\sigma$ est représentée sur la figure 1. Après une phase de 2 jours pendant laquelle les perturbations initiales sont lissées, le système commence à s'auto-organiser. L'amplitude des perturbations augmente exponentiellement et le taux de croissance est constant. C'est la phase linéaire. Elle se produit entre le $6^{\text {ème }}$ et le $8^{\text {ème }}$ jour (figure $1 \mathrm{~h}$, Phase I). Ensuite, la croissance des instabilités sature (figure 1h, phase II) et enfin un équilibre (dynamique) est atteint, c'est ce que l'on appelle le régime à amplitude finie (figure 1, Phase III). Les caractéristiques des barres en croissant n'évoluent plus de manière significative jusqu'à la fin de la simulation préliminaire ( $t=116$ jours). A la fin de la simulation préliminaire, 12 barres en croissant sont développées. La longueur d'onde moyenne des figures rythmiques $\lambda_{\text {moy }}$ est de $167 \mathrm{~m}$. La norme euclidienne des perturbations est de l'ordre de $15 \mathrm{~cm}$. La figure $2 \mathrm{~b}$ représente la bathymétrie au début et à la fin de la simulation préliminaire.

a)

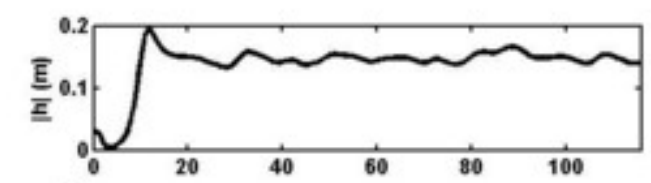

b)

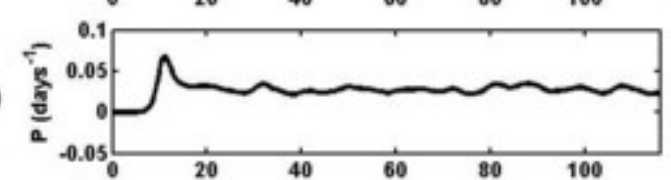

c)

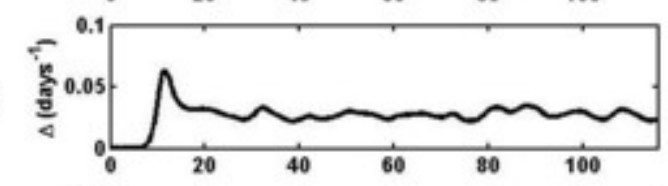

d)

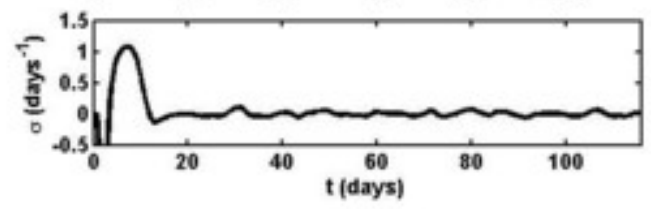

e)
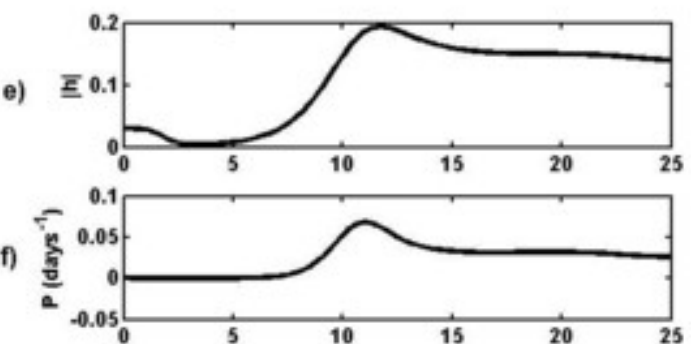

g)

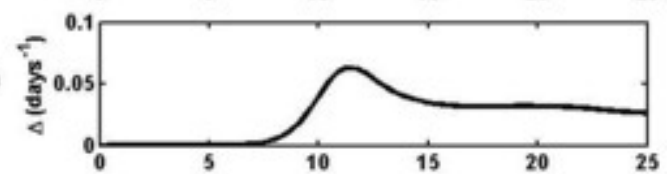

h)

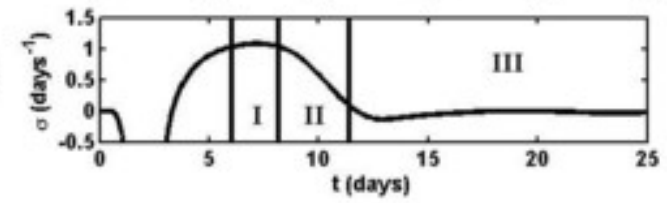

Figure 1. Evolution temporelle de $\|h\|(a)$, de $P(b)$, de $\Delta$ (c) et de $\sigma(d)$ pendant la simulation préliminaire $\left(\theta=0^{\circ}\right)$. Les graphes (e, $f$, g et $h$ ) sont des "zooms" temporels. 

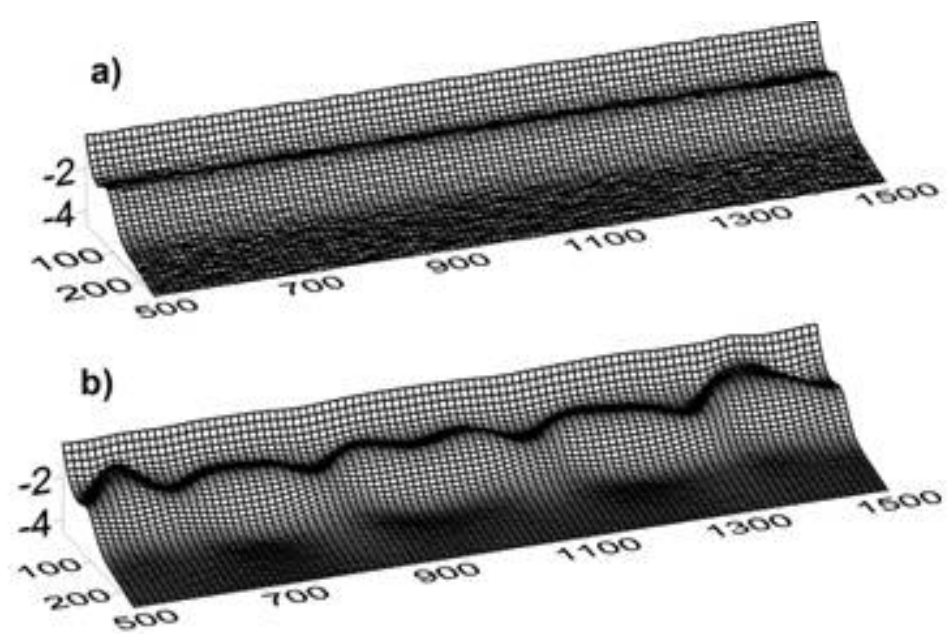

Figure 2. Visualisation en $3 D$ de la bathymétrie au début (a) et à la fin (b) de la simulation préliminaire ( $t=0$ et $t=116$ jours respectivement).

3.2 Réponse des barres en croissant en présence de courant longshore (vagues obliques) Pour simuler la réponse de figures rythmiques préexistantes à un courant longshore, nous imposons à présent des vagues obliques. La hauteur et la période des vagues ne sont pas modifiées. La bathymétrie de départ pour ces simulations est celle obtenue à la fin de la simulation préliminaire (figure $2 \mathrm{~b}$ ). En pratique, la simulation préliminaire est prolongée en modifiant l'incidence des vagues $\theta$ à partir de $t=116$ jours. Si $t<116$ jours alors $\theta=0^{\circ}$; si $116<t<119$ jours alors $\theta$ passe de $0^{\circ}$ à la valeur souhaitée $\left(0^{\circ}, 3,5^{\circ}, 7\right.$ ou $\left.13,5^{\circ}\right)$; si $t>119$ jours alors, selon les cas, $\theta=0^{\circ}, 3,5^{\circ}, 7$ ou $13,5^{\circ}$.

La figure 3 représente l'évolution temporelle des variables $\|h\|, P$, $\Delta$, et $\sigma$. Le cas $\theta=0^{\circ}$ est une prolongation de la simulation préliminaire. L'équilibre dynamique est maintenu jusqu'à la fin de la simulation. Le taux de croissance reste proche de zéro (figure $3 \mathrm{~d}$ ) et les caractéristiques des barres en croissant évoluent peu : la norme des perturbations $\|h\|$ se maintient autour de $15 \mathrm{~cm}$ (figure $3 \mathrm{a}$ ) et la longueur d'onde moyenne $\lambda_{\text {moy }}$ est stabilisée à $167 \mathrm{~m}$. La géométrie des barres en croissant à la fin de la simulation $(t=289$ jours) est représentée sur la Figure $4 \mathrm{a}$, elle est comparable à la géométrie des barres en croissant à $t=116$ jours (figure $2 \mathrm{~b}$ ).

Pour les cas $\theta=3,5^{\circ}, 7^{\circ}$ et $13,5^{\circ}$, la figure 3 montre que le changement de direction des vagues à la fin de la simulation préliminaire (à partir de $t=116$ jours) modifie fortement le comportement du système. Dès que $\theta$ augmente, $\|h\|, P, \Delta$, et $\sigma$ diminuent sensiblement. Cette diminution est d'autant plus rapide que la valeur de $\theta$ est élevée. Le comportement du système est différent pour $\theta=3,5^{\circ}$ d'une part et pour $\theta=7^{\circ}$ ou $13,5^{\circ}$ d'autre part. 
a)

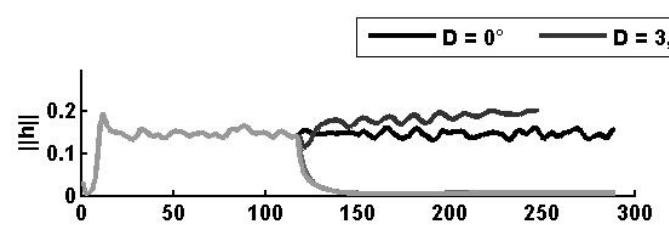

b)

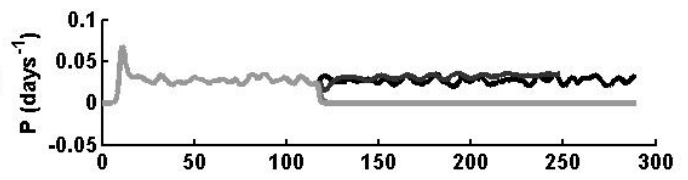

c)

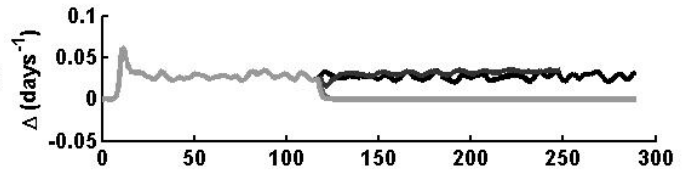

d)

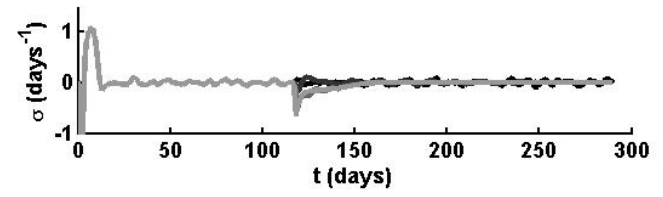

e)

$D=7^{\circ} \longrightarrow \mathrm{D}=13,5^{\circ}$
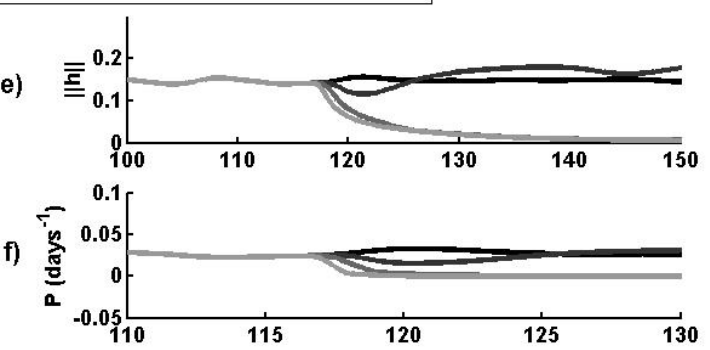

g)

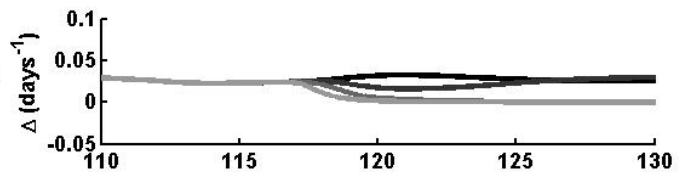

h)

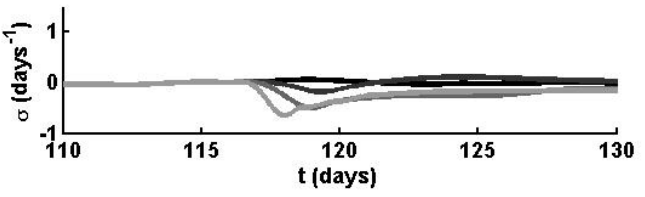

Figure 3. Evolution temporelle de $\|h\|(a)$, de $P(b)$, de $\Delta(c)$ et de $\sigma(d)$ pendant $l$ 'ensemble de la simulation. Les graphes (e, $f$, g et h) sont des "zooms" temporels.
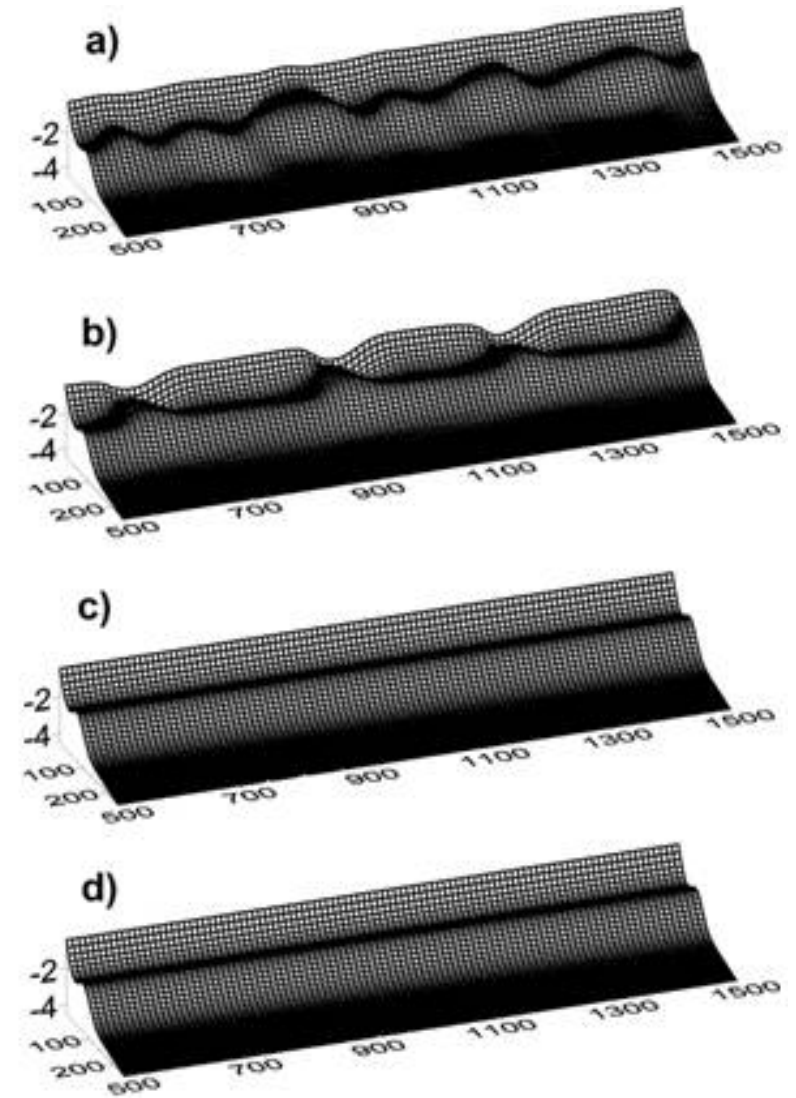

Figure 4. Visualisation en $3 D$ de la bathymétrie à la fin de la simulation ( $t=289$ jours) pour les cas $\theta=0^{\circ}(a), \theta=3,5^{\circ}(b), \theta=7^{\circ}$ (c) ou $\theta=13,5^{\circ}\left(\right.$ d). Pour le cas $\theta=3,5^{\circ}$, les résultats sont extraits à $t=174$ jours (explosion du modèle à $t=248$ jours). 
Dans le cas $\theta=3,5^{\circ}$, au bout de quelques jours, la production d'instabilités reprend (figures $3 \mathrm{~b}$ et $3 \mathrm{f}$ ) et le taux de croissance redevient positif (figure $3 \mathrm{~d}$ et $3 \mathrm{~h}$ ). La norme des perturbations augmente à nouveau progressivement jusqu'à atteindre une valeur proche de $20 \mathrm{~cm}$ (figure 3a) quand le modèle "explose" à $t=248$ jours (l'explosion du modèle est liée au fait que les effets de pente ne suffisent pas pour compenser la croissance des instabilités). La bathymétrie à $t=174$ jours est représentée sur la figure 4b. Cette figure montre que les 12 barres en croissant préexistantes (figure 2b) ont fusionné pour former des barres de plus grande dimension (figure $4 \mathrm{~b}$ ). La longueur d'onde passe de $167 \mathrm{~m}$ (12 croissants en $2 \mathrm{~km})$ à $333 \mathrm{~m}$ ( 6 croissants en $2 \mathrm{~km})$.

Pour les cas $\theta=7$ et $13,5^{\circ}$, la production d'instabilités $P$ diminue jusqu'à la fin de la simulation (Figures $3 \mathrm{~b}$ et $3 \mathrm{f}$ ). Le taux de croissance $\sigma$ reste négatif (figures $3 \mathrm{~d}$ et $3 \mathrm{~h}$ ) car $P<\Delta$. Les perturbations sont lissées progressivement (figures $3 \mathrm{a}$ et $3 \mathrm{~d}$ ) et le système revient à une configuration proche de la configuration initiale (figures $4 \mathrm{c}$ et $4 \mathrm{~d}$ ).

\section{Discussion}

L'influence de l'orientation des vagues sur la formation de figures rythmiques a déjà été analysée. Par exemple, CALVETE et al. (2005), GARNIER et al. (2009) ou THIEBOT et al. (2012) ont montré qu'en partant de barres rectilignes, plus l'incidence des vagues au large est importante plus les barres en croissant formées sont allongées dans la direction parallèle à la plage. Il semble que la tendance soit comparable dans les configurations testées (configuration initiale comportant des barres en croissants développées) : dans notre étude, quand $\theta$ augmente de $0^{\circ}$ à $3,5^{\circ}$, la longueur d'onde des croissants préexistants passe de 167 à $333 \mathrm{~m}$. GARNIER et al. (2009) ou THIEBOT et al. (2012) ont également montré que, pour la formation des figures rythmiques (évolution associée aux séquences down-state), à partir d'une certaine incidence le taux de croissance des instabilités est tellement faible que le système reste stable (aucune instabilité ne se développe, les barres restent rectilignes). Ici encore, il est possible de faire un parallèle avec les configurations testées : pour les cas $\theta=7^{\circ}$ et $13,5^{\circ}$, la bathymétrie finale est également une configuration sans instabilité. Dans les configurations testées, tout se passe comme si le système se réadaptait aux conditions de vagues imposées, qu'il y ait des croissants préexistants ou pas au début de la simulation. Enfin, l'analyse globale montre que lorsque le système est en équilibre dynamique (phase III sur la figure 1), les termes de production et de diffusion se compensent (i.e. $P \approx \Delta$ et $\sigma \approx 0$ ). La modification de l'orientation des vagues (à partir de $t=116$ jours) rompt cet équilibre (figure 3). Il y a à la fois une diminution de la production et de la diffusion (figure $3 \mathrm{f}$ et $3 \mathrm{~g}$ ). Comme la diminution de $P$ est plus rapide que celle de $\Delta$ (figures $3 \mathrm{f}$ et $3 \mathrm{~g}$ ), $\sigma$ devient négatif (figure $3 \mathrm{~h}$ ) et l'amplitude des perturbations se réduit fortement. 


\section{XII ${ }^{\text {èmes }}$ Journées Nationales Génie Côtier - Génie Civil \\ Cherbourg, 12-14 juin 2012}

\section{Conclusion}

En travaillant sur des configurations idéalisées, la présente étude confirme les observations selon lesquelles le courant longshore (ou l'obliquité des vagues) peut jouer un rôle important dans la disparition des figures rythmiques (évolution observée pendant les séquences "up-state"). En effet, dans les configurations testées, il est possible de reproduire l'apparition puis la disparition de barres en croissant rien qu'en modifiant la direction des vagues. Dans la présente étude, les croissants disparaissent pour des incidences supérieures ou égales à $7^{\circ}$ au niveau de la crête de la barre. La disparition des instabilités s'explique par une diminution rapide de la production d'instabilité. Un travail complémentaire est nécessaire pour comprendre les raisons de la diminution de la production d'instabilités et pour généraliser ces résultats à l'ensemble des plages comportant des barres en croissant. Il s'agira de tester d'autres profils, d'autres conditions de vagues, d'étudier l'influence de la marée ou de courants induits par le vent... Enfin, certaines observations montrent que les barres en croissant peuvent disparaitre en présence de vagues énergétiques et frontales. Bien que le courant longshore joue un rôle important dans les séquences up-state, il n'est donc pas l'unique mécanisme responsable cette évolution morphodynamique.

\section{Références bibliographiques}

CABALlERIA M., COCO G., FALQUÉS A., HUNTLEY D.A. (2002). Selforganization mechanisms for the formation of nearshore crescentic and transverse sand bars. J. Fluid Mech., 465, pp 379-410. doi:10.1017/S002211200200112X

CALVETE D., DODD N., FALQUÉS A., VAN LEEUWEN S.M. (2005). Morphological development of rip channel systems: normal and near normal wave incidence. J. Geophys. Res., 110, C10006, doi:10.1029/2004JC002803

CASTELLE B., RUESSINK B.G. (2011) Modeling formation and subsequent nonlinear evolution of rip channels: Time-varying versus time-invariant wave forcing, Journal of Geophysical Research, 116, No. F4, F04008.

FALQUÉS A, COCO G, HUNTLEY DA (2000). A mechanism for the generation of wave driven rhythmic patterns in the surf zone. J.Geophys.Res. 105(C10), pp 24071-24087.

GARNIER R., CALVETE D., FALQUÉS A., CABALLERIA M (2006). Generation and non linear evolution of shore-oblique/transverse bars. J. Fluid Mech., 567, pp 327-360. doi:10.1017/S0022112006002126

GARNIER R., CALVETE D., FALQUÉS A., DODD N. (2008). Modelling the formation and the long-term behaviour of rip channel systems from the deformation of a longshore bar. J. Geophys. Res., 113, C07053, doi:10.1029/2007JC004632

GARNIER R., DODD N., FALQUÉS A., CALVETE D. (2009). A mechanism inhibiting rip channel formation for oblique waves. Proceedings of Coastal Dynamics, 
Thème 2 - Dynamique sédimentaire

Impacts of Human Activities on Dynamic Coastal Processes, pp 1-13. doi:10.1142/9789814282475_0039

GARNIER R., DODD N., FALQUES A., CALVETE D (2010). Mechanisms controlling crescentic bar amplitude. J. Geophys. Res., 115,F02007. doi:10.1029/2009JF001407

MEI C.C. (1989) The Applied Dynamics of Ocean Surface Waves. Advanced series on Ocean Engineering. vol. 1. World Scientific.

PRICE T.D., RUESSINK B.G. (2011). State dynamics of a double sandbar system. Continental Shelf Research, 31(6), pp 659-674. doi:10.1016/j.csr.2010.12.018

SOULSBY R.L. (1997). Dynamics of marine sand: A manual for practical applications. Thomas Telford Publications, London, $249 \mathrm{p}$.

THIEBOT J., IDIER D., GARNIER R., FALQUES A., RUESSINK B.G. (2012). The influence of wave direction on the morphological response of a double sandbar system. Continental Shelf Research, 32, pp 71-85. doi:10.1016/j.csr.2011.10.014

WRIGHT L.D., SHORT A.D. (1984) Morphodynamic variability of beaches and surf zones, a synthesis. Marine Geology, 56, pp 92-118. doi:10.1016/0025-3227(84)90008-2

YU J., SLINN D.N. (2003). Effects of wave-current interaction on rip-currents, Journal of Geophysical Research, 108, C33088, doi:10.1029/2001JC001105 